\title{
The average shape of the closed trefoil knot fluctuating on a floppy rope
}

\author{
Marcin Modlinski ${ }^{a}$, Sylwester Przybyl, and Piotr Pieranski \\ Department of Computational Physics, Poznan University of Technology, Nieszawska 13A, 60-965 Poznan, Poland
}

Received 8 August 2012

Published online: 14 May 2013

(c) The Author(s) 2013. This article is published with open access at Springerlink.com

\begin{abstract}
The average shape of the trefoil knot tied on a floppy, hard rope subject to thermal fluctuations has been determined. The fluctuations of the shape of knots were performed by random bending. As a result of the changing shape procedure large sets of deformed conformations of the initial knot were obtained. Afterwards, these sets were subject to the shape-fitting procedure. It has been found that the conformation is different from the ideal conformation of the knot.
\end{abstract}

\section{Introduction}

Suppose a closed knot has been tied on a piece of a rope subject to thermal fluctuations. The rope has such a length that thermal fluctuations constantly change the shape of the knot. In terms of the physics of polymers, this model describes a knotted ring polymer submerged in a thermal bath. An essential question arises: What is the average shape of the fluctuating knot? The answer to this question depends both on the physical properties of the rope in which the knot is tied and on what we mean by "the average shape".

In the simplest case studied by Millet et al., see ref. [1], the "rope" is just six infinitely thin and stiff segments of equal length connected with each other into a hexagonal trefoil knot. The segments are connected in such a manner that no energy is needed to change the angles of the hexagon. Do the thermal fluctuations of such hexagonal knots cause them to explore the whole accessible space of conformations? Obviously, the space is different when the hexagon is knotted and when it is unknotted. Steric hindrances stemming from the impenetrability of the stiff segments do not allow the hexagon to change its topology. Thus, when it is initially unknotted it remains unknotted all the time; when it is knotted, it remains knotted all the time. Let us remind here that the knot in question is the trefoil. Results of the study described in ref. [1] concerning the knotted hexagons are relevant to the results of our study, thus, let us concentrate on them. The exploration of the space of the knotted conformations was achieved in ref. [1], not by modifying the shape of the hexagon but by a multiple creation using the hedgehog method. It is important to remember that the trefoil knot is chiral, i.e.

\footnotetext{
${ }^{a}$ e-mail: Marcin.Modlinski@doctorate.put.poznan.pl
}

it has left- and right-handed forms. Thermal fluctuations of the knot tied on an impenetrable rope are not able to change its chirality type: for instance, a right-handed trefoil will always remain right-handed. Hexagonal righthanded trefoil knots created via the hedgehog procedure were translated in such a manner that their centre of mass was shifted to the origin of the $(x, y, z)$ Cartesian reference frame. Then the knots were apropriately rotated. The aim was to orient eigenvectors of their gyration tensor along the axes of the reference frame. The longest gyration tensor eigenvector was oriented always along the $x$-axis. The middle eigenvector of the tensor was oriented along the $y$-axis. Consequently, since the eigenvectors of the tensor are orthogonal, the shortest gyration tensor eigenvector was oriented along the $z$-axis of the reference frame. The essential detail of the procedure was what the authors call symmetry breaking alignment, see [1]. Generating a large number of knots oriented in such a manner the authors obtained a 3D cloud of points and they analysed its spatial density. The analysis, see fig. 4(b) in ref. [1] reveals the chiral nature of the knot.

The question concerning the average shape of a fluctuating knot, for knots tied on an elastic rope, has been recently asked and answered by Saka and Takano [2]. The answer that they provide depends not only on the details of the interaction potentials defining the elastic properties of the model rope they used, but also on the temperature of the thermal bath within which the simulated knot was submerged. Saka and Takano performed their simulations for a particular interaction potential and at a particular temperature. It is difficult to draw more general conclusions from a single such study. There exists, however, a particular type of model rope for which the temperature of the thermal bath becomes irrelevant. This model rope, called perfect rope, being perfectly floppy is at the same 
time perfectly hard [3]. The rope is perfectly hard if the shapes of its perpendicular sections do not change even when the rope is squeezed. The sections of the perfect rope are circular. They can be thus seen as hard disks. Being hard they not only cannot be deformed but also they are not allowed to overlap. It is essential to notice that overlaps of the section disks can occur not only when two axially distant points of the rope are brought too close together, i.e. to a distance smaller than the diameter $D$ of the rope, but also when the rope is bent too much. In the first case the doubly critical self-distance becomes smaller than $D$. In the second case the local curvature becomes larger than $2 / D=1 / R$, where $R=D / 2$ is the radius of the circular sections of the rope. The perfect rope is also perfectly floppy: as long as the local curvature of the rope axis is smaller than $1 / R$, bending it costs no energy. Bending the perfect rope above this limit is prohibited. The above-described physical properties of the perfect rope imply that the potential energy of the knot tied on such a rope is always equal to zero. As a result, all legitimate conformations of the knot are equally probable. The average shape of the knot tied on the perfect rope depends solely on the length of the rope in which it has been tied, but it is temperature independent: a higher temperature makes the knot fluctuate faster, but the space of its accessible conformations and, thus, its average shape remain the same. Knots tied on the perfect rope can be seen as reference points in the physical theory of thick knots tied on ropes with various physical properties. It is our aim to find out, quantitatively, how their average shape changes with the length of the rope on which they have been tied.

As Saka and Takano have found, the trefoil knot tied on the elastic rope subject to thermal fluctuations becomes asymmetrical: the threefold symmetry characteristic of its tight conformation $[4,5]$ vanishes - one of its three foils becomes larger than the two other foils. One can arrive at this conclusion through an analysis of the results of studies which localise the knotted region within knots tied on long polymer filaments. It has been convincingly demonstrated that thermal fluctuations "squeeze" the knot into knotted polymer rings [6-12]. The knots considered in those papers are either based on the lattice model or regular chains polymer in two or three dimensions. Interesting study was carried out for knots tied on the granular chains [13] and knots tied on charged polymers [14]. Thus, one can predict that in the case of the closed trefoil knot tied on a long piece of a floppy rope one of its foils should be on average longer than the two other foils. It is interesting to ask if this behaviour becomes visible also in the almost tight trefoil knots, i.e. if the almost tight knots lose the threefold symmetry axis present in the tightest trefoil. We will confirm the asymmetry of the average shape but also quantitatively determine the geometrical parameters of the changing average shape, in particular its curvature and torsion profiles. We will give a simple criterion allowing one to identify all three foils of the knot. This has allowed us to precisely measure their lengths and determine how the lengths change with the increasing length of the rope in which the knot had been tied.

\section{The simulation method}

\subsection{Discrete representation of knots tied on the perfect rope}

Knots tied on the perfect rope are smooth: their tangent vector is continuous. For obvious reasons, in numerical simulations we deal with polygonal knots for which the tangent vector becomes discontinuous at those of its vertices which are the meeting points of non-collinear segments. The relation between the polygonal knots that we numerically process and the smooth knots, which the polygonal knots aim to represent, is thus essential. In what follows we shall discuss it using as an example the tightest of all trefoil knots tied on the perfect rope of radius $R=1$ described parametrically by a set of three functions. Construction details of the polygonal knots $K_{\text {poly }}$ are described in previous publications [3]. Using the Rawdon method it is possible to inscribe a curvilinear knot into a polygonal knot [5].

To make the considerations presented below compatible with the considerations described by Saka and Takano, we decided to use their notation. A continuous closed knot $K$ is a self-avoiding curve in the 3 -dimensional space. The curve is represented in numerical simulations by the set of $N$ equidistant points $P_{i}, i=0,1, \ldots, N-1$, which can be seen as vertices of an equilateral polygon $K_{\text {poly }}$. The polygon representing in our simulations knot $K$ will be denoted by $C$. Obviously, for a given continuous knot there are an infinite number of polygonal representations, depending on where we place the first vertex in the knot. When starting a simulation we just choose one of them at random. The results of the study do not depend on this choice. In a given Cartesian coordinate frame each of the vertices of $C$ is indicated by a position vector $\mathbf{R}_{i}, i=0,1, \ldots, N-1$. Thus, any conformation $C$ of the simulated knot is represented by the set of $N$ vectors,

$$
C=\left\{\mathbf{R}_{0}, \mathbf{R}_{1}, \mathbf{R}_{2}, \ldots, \mathbf{R}_{N-1}\right\} .
$$

In view of the assumed equilaterality of $C$, distances between consecutive points are identical,

$$
\left\|\mathbf{R}_{i+1}-\mathbf{R}_{i}\right\|=d l, i=0,1,2, \ldots, N-1 .
$$

Since the knot is closed,

$$
\mathbf{R}_{N} \equiv \mathbf{R}_{0}
$$

The polygonal length of the knot,

$$
L(0)_{\text {poly }}=N d l \text {. }
$$

All procedures, except the final averaging procedure, that we apply in our simulations keep this value constant. As a result, all conformations of the fluctuating knot obtained from our simulation are equilateral polygons of constant length. Let us emphasise here, that although, for the sake of brevity, we refer to $C$ as the polygonal knot, we have in mind a polygonal knot whose vertices are surrounded by hard spheres of unit radius. This is essential, since the 
hard spheres surrounding the vertices of $C$ limit in an essential manner the freedom of its fluctuations. Axially distant vertices of such a knot cannot come closer to each other than $D=2 R$ and the bending angles within the knot cannot be larger than

$$
\Theta_{\max }=2 \arcsin \left(\frac{d l}{2 R}\right) .
$$

Let us describe in more detail the knots the fluctuations of which we have simulated. There were 24 of them. All of them were trefoils, but they differed in their length. Thinking about trefoils tied on the perfect rope we come to the conclusion that there exists a particular trefoil that can be tied on a piece of the perfect rope of a minimum length. The tightest trefoil is our starting point. To be more precise, our starting point is the tightest polygonal knot whose vertices are surrounded by spheres of unit radius. The knot is tight, i.e. the spheres surrounding its vertices stay in touch. As a result, its shape is well defined. The knot is locked; it has no freedom to fluctuate. To allow the fluctuations the knot must be made longer. We used polygonal trefoils of 24 different lengths $L(0)_{\text {poly }}>L_{\text {tight }}$, where $L_{\text {tight }}$ is the length of the tightest polygonal knot. For simplicity, $L$ denotes in what follows the polygonal length $L_{\text {poly }}$ of the knots. To keep the precision of the discretisation of all analysed knots at the same level, the number $N$ of the vertices was appropriately increased with the length $L(0)_{\text {poly }}$ of the simulated knot. As a result, the segment length $d l$ in all simulated knots has always almost the same value. The tightest trefoil knot had its centre of mass in the origin of the $(x, y, z)$ reference frame and its threefold axis was oriented along the $z$-axis.

\subsection{Elementary motions changing the shape of the knot}

What we are interested in are not the thermal motions of the knot as a whole, i.e. its Brownian-motion-like translations and rotations, but the thermal fluctuations of its shape. Consequently, the elementary motions applied in the simulation should change the shape of the knot but not either translate or rotate it.

Let $C(0)$ be one the 24 initial conformations of the processed knot. Obviously, as explained above, its length $L(0)>L_{\text {tight }}$. In what follows, the position of its vertices will be denoted as $R_{i}(0)$. The conformation was subject to a sequence of $n$ elementary motions aimed at changing its shape. Each of the elementary motions was performed as follows:

1) A pair of vertices $(i, j)$, where $|j-i|>1$, was chosen at random. The pair of vertices was dividing the knot into two (as a rule, unequal) pieces and it was defining an axis $O$ passing through both of them (see fig. 1).

2) The smaller piece of the knot located between the chosen vertices was rotated around the axis $O$ by a small angle $\alpha$ chosen at random from the interval $\left(0, \alpha_{\max }\right)$. The sense of the rotation depended on the order of the chosen indexes $i, j, e . g$. , choices $(2,10)$ and $(10,2)$ meant different senses of the rotation.

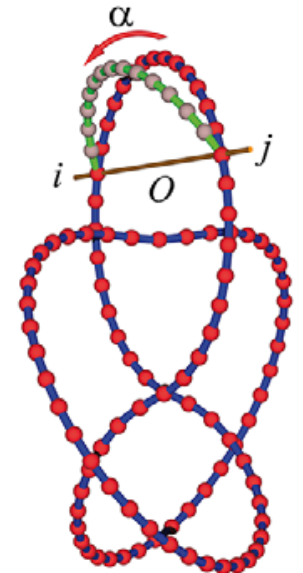

Fig. 1. The idea of the bending procedure - the most essential element of elementary moves changing the shape of the fluctuating knot.

The elementary move defined above was accepted when the conformation obtained by its application was free from overlaps, where by overlaps we understand both the situations when two of the unit spheres surrounding axially distant vertices of the knot become closer than $D=2 R$ and when any of the bending angles becomes larger than $\Theta_{\max }$. When either of the two conditions was broken, the elementary move was cancelled and a new one was generated. The maximum rotation angle, $\alpha_{\max }$, was set to such an experimentally determined value, at which about $80 \%$ of elementary moves were becoming accepted. Let us describe now how from the initial conformation $C(0)$ of the fluctuating knot its next conformation, $C(1)$ was obtained.

There exists a weak point in our work: in contrast to the hedgehog algorithm used in ref. [1], we are not able to prove that the elementary moves which we are using are ergodic.

The sequence of $n_{\mathrm{b}}=1000$ of the bending moves performed, according to the above-described procedure, changes the shape of the initial conformation leaving the position of its centre of mass intact. Final positions of the vertices of the knot are denoted by $\mathbf{R}_{i}(1)$. Thus, vectors $\mathbf{R}_{i}(1), i=0,1, \ldots, N-1$ define the new conformation of the knot; we denote it by $C(1)$. Consecutive conformations $C(2), C(3), \ldots, C(M)$ are obtained in the same manner, i.e. the next conformation is obtained from the previous one by subjecting the latter to the sequence of $n_{\mathrm{b}}$ shape-changing motions and the appropriate correction of the position of the centre of mass. The relation between consecutive conformations of the sequence can be thus written as

$$
C(m+1)=\mathcal{B}(C(m)),
$$

where $\mathcal{B}$ denotes the effective procedure that randomly changes the knot shape without changing the position of its centre of mass. The sequence $C(1), C(2), \ldots, C(M)$ of the recorded conformations of the fluctuating knot we denote by $\mathcal{C}$. Thus,

$$
\mathcal{C}=\{C(1), C(2), \ldots, C(M)\} .
$$




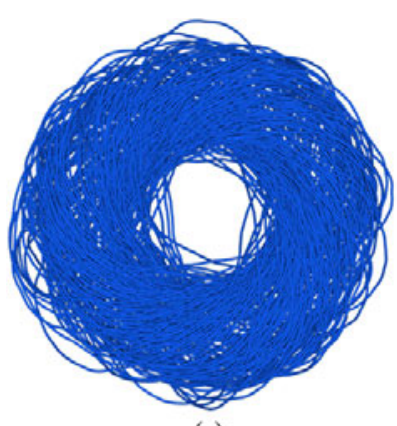

(a) (b)

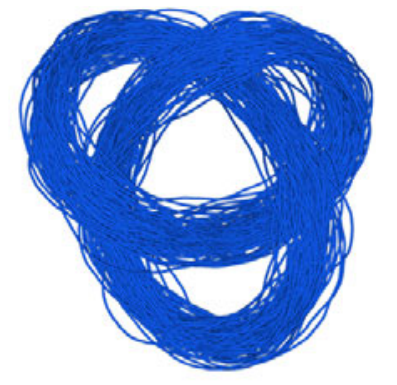

Fig. 2. Conformations of the fluctuating trefoil knot before (a) and after (b) the shape fitting procedure. Picture (a) presents 100 conformations from the initial set $\mathcal{C}$, while picture (b) shows the same conformations after the appropriate re-orientations determined via the shape-fitting procedure. The appropriately re-oriented and re-indexed conformations are gathered in set $\mathcal{C}^{\prime}$. It is the averaging of the positions of their vertices that provides the positions of the average shape conformation $C^{\text {av }}$. It is obvious that the average shape conformation cannot be found by the direct averaging of the vertex positions of original conformations shown in (a), but it can be found using conformations shown in (b).

The problem that we now face is: how to determine the average shape of all conformations gathered in $\mathcal{C}$ ? The problem is by no means trivial. The method that we have arrived at is, as we have found, analogous to that applied by Saka and Takano [2]. It consists of two steps. In the first step, the conformations gathered in $\mathcal{C}$ are fitted to each other via appropriate rotations and re-indexing, so that the mean square distance between all the conformations reaches its minimum. The fitting procedure is similar to the procedure applied by Amzallag et al. [15]. The conformations obtained after the application of this fitting procedure are gathered in a new set denoted by $\mathcal{C}^{\prime}$. In the last step the average of all conformations belonging to $\mathcal{C}^{\prime}$ is determined. It is this shape that can be seen as the average shape of the fluctuating knot.

The efficiency of the shape-fitting procedure is illustrated in fig. 2. On the left we present 100 conformations belonging to the initial set $\mathcal{C}$. As one can see their average shape is by no means visible here. However, as shown in fig. 2(b), when the same conformations are appropriately re-oriented (the re-oriented conformations were taken from set $\mathcal{C}^{\prime}$ ) the average shape of the fluctuating knot becomes well visible even to the naked eye. In particular, one can easily see that one of the knot foils is larger than the two other foils.

\subsection{The average shape of the fluctuating trefoil knot}

The trefoil knots of 24 lengths have been subject to fluctuations. Initial conformations of the knots were obtained by an appropriate scaling of the tightest knot, so that their lengths $L(0)$ were equal,

$$
L(0)=(\varepsilon+1) L_{\text {tight }},
$$

where $0<\varepsilon \leq 1$ is the scaling factor and $L_{\text {tight }}$ is the length of the tightest trefoil knot, $L_{\text {tight }}=32.74$. Let us
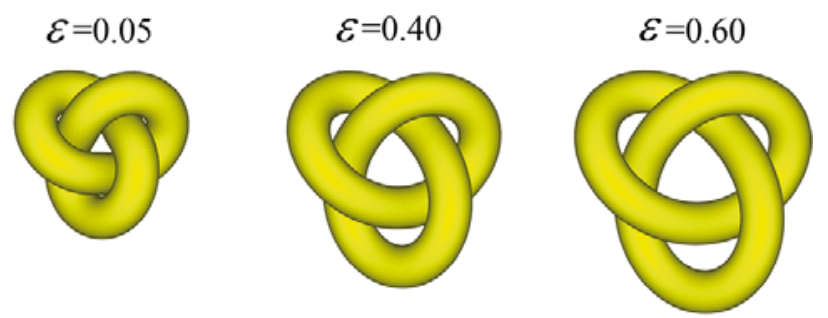

$\mathcal{E}=0.80$
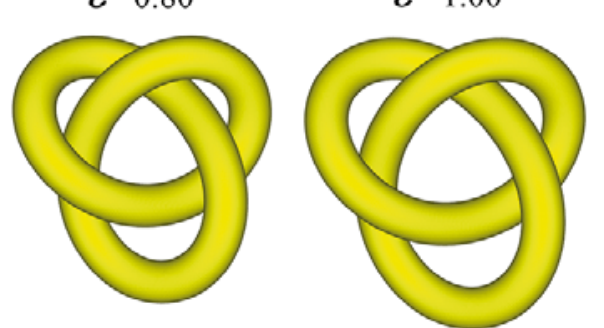

Fig. 3. Five of the 24 average shape conformations found for the thermally fluctuating trefoil knots tied on the perfect rope of an increasing length.

recall that making the knot longer, we were appropriately increasing the number $N$ of its vertices, so that the length $d l$ of its segments (distances between consecutive vertices) was kept approximately constant.

Summarising the whole procedure, let us state that each of the 24 knots was subject to $n=3 \cdot 10^{7}$ elementary bending moves. Conformations of the fluctuating knots were recorded every $n_{\mathrm{b}}=10^{3}$ move. Thus, for each of the knots, a set $\mathcal{C}=\{C(1), C(2), \ldots, C(M)\}$ of $M=3 \cdot 10^{4}$ conformations was created. Subsequently, the conformations were rotated and re-indexed in such a manner that they became as close to each other as possible (fig. 2). The set of the re-oriented and re-indexed conformations was denoted by $\mathcal{C}^{\prime}$. Only then, the average conformation of the fluctuating knot was determined.

Figure 3 presents the average shapes of 5 of the 24 trefoil knots subject to fluctuations. It is clearly seen that as the fluctuating trefoil knot becomes longer its average shape changes in a well-defined manner: the lengths of all three foils of the knot become different. In general, the fluctuating trefoil knot loses in its average conformation all of its symmetry elements, which are characteristic to the tightest conformation.

\subsection{Evolution of the lengths of the foils}

Let $L(0)$ be the length of the trefoil knot subject to fluctuations. Let us remind that $L(0)$ is not the length of the tightest trefoil knot, but the length of the tightest trefoil knot scaled up by factor $\varepsilon+1$. The initial knot is equilateral. The bending moves used to simulate the thermal fluctuations do not change its basic properties, i.e. all conformations gathered in the $\mathcal{C}$ set are also equilateral and their length equals $L(0)$. Obviously, all of the abovedescribed rotations and re-indexing aimed at making the conformations as congruent to each other as possible do not change the properties either. However, this particular conformation that defines the average shape of the fluctuating knot, found by calculating average positions of the 


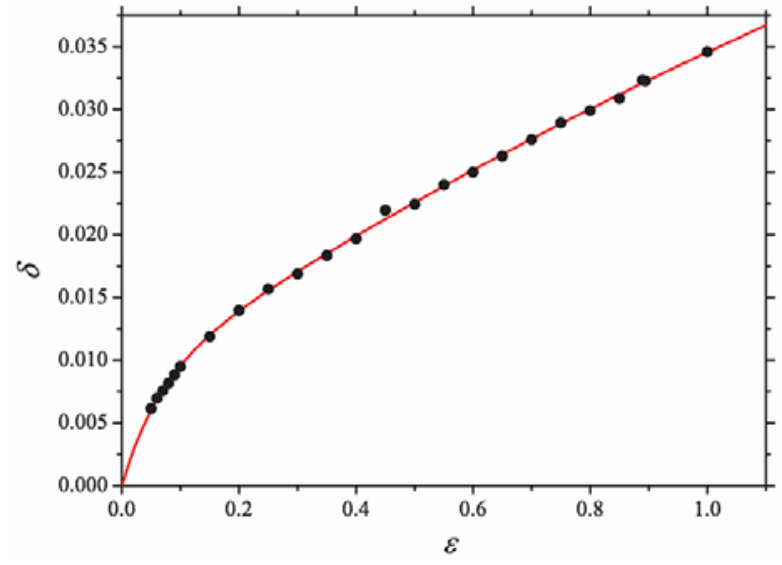

Fig. 4. Dependence of the shortening parameter $\delta$ on the excess length parameter $\varepsilon$. See text.

vertices of all conformations belonging to the $\mathcal{C}^{\prime}$ set obtained at the end of the congruence seeking procedure, is no more equilateral and its length $L^{\text {av }}$ is smaller than $L(0)$. Let us define a variable that reflects this shortening,

$$
\delta=\frac{L(0)-L^{\mathrm{av}}}{L(0)} .
$$

Figure 4 presents the dependence of this variable on the value of the $\varepsilon$ parameter that describes the amount of the freedom of the knot to fluctuate.

$L^{\text {av }}$ is the length of the average shape conformation of the fluctuating knot. It is interesting to determine how the length is divided between the tree foils of the knot. Obviously, to divide $L^{\text {av }}$ into the three parts that would correspond to the three foils, one needs to define an algorithm of this division. The most natural manner is to find those pairs of points within the knot at which the self-distance of the knotted rope reaches its minima. There are three such pairs: $\left(P_{1}, P_{2}\right),\left(P_{3}, P_{4}\right),\left(P_{5}, P_{6}\right)$. The arc length distances $l_{1}^{\text {av }}, l_{2}^{\text {av }}, l_{3}^{\text {av }}$ between points belonging to the pairs can be seen as the lengths of three foils $\left(F_{1}^{\mathrm{av}}, F_{2}^{\mathrm{av}}, F_{3}^{\mathrm{av}}\right)$ of the analysed knot. If the average shape conformation had the threefold symmetry characteristic of the tightest knot, all the foils would be of the same length. Looking at fig. 5 we see that this is certainly not the case: all three foils have a different length.

\subsection{Evolution of the curvature and torsion profiles}

The curvature profile of the tightest trefoil knot was presented and discussed in ref. [5]. The most interesting landmarks of the profile were the double peaks, perfectly flat at their tops because they were cut off by the maximum curvature limit. For knots tied on hard, circular ropes the maximum curvature $\kappa_{\max }=2 / D$, where $D$ is the diameter of the rope. Peaks within each of the double peaks were separated by a distinct valley. See fig. 12 in ref. [5]. Making the knot loose changes significantly this curvature profile. Already a very small departure, measured here by the value of the $\varepsilon$ parameter, makes the double peaks disappear. The valley, however, remains. See fig. 6. As a result, the curvature profile of the average shape conformation
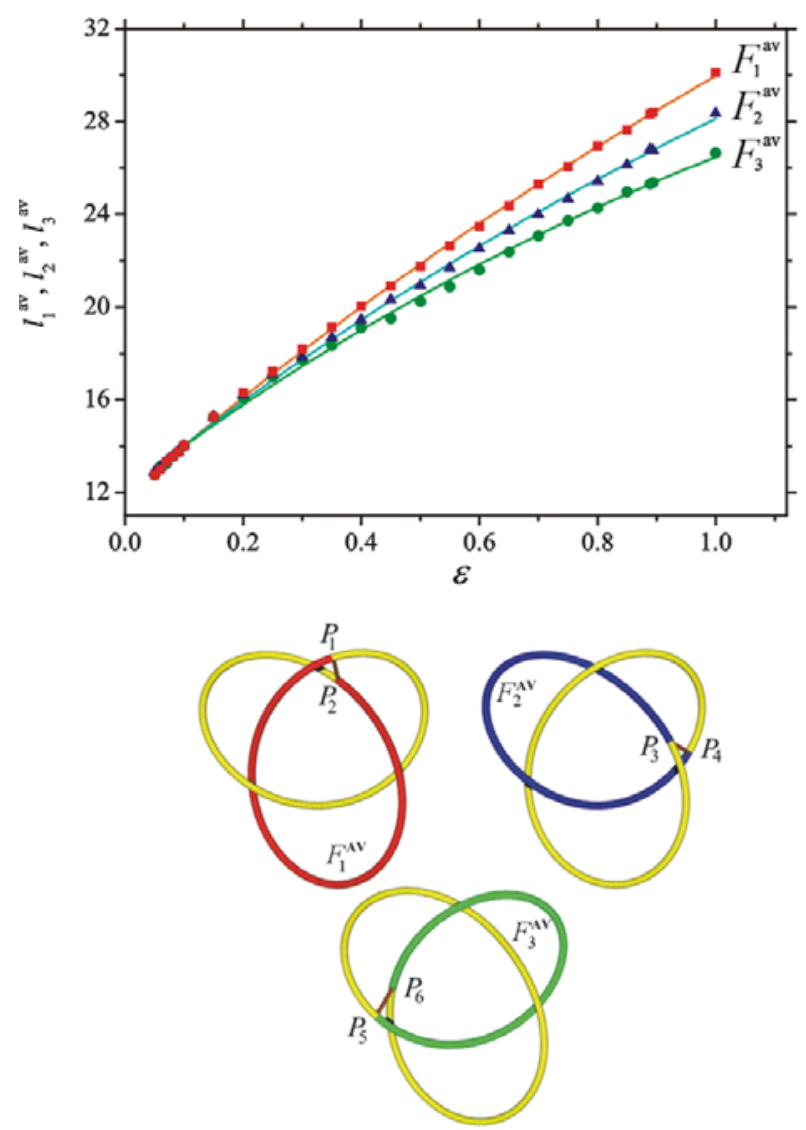

Fig. 5. Lengths of the knot foils versus the elongation parameter $\varepsilon$. At $\varepsilon=0$ the knot is closely packed and all three leaves are of identical length.

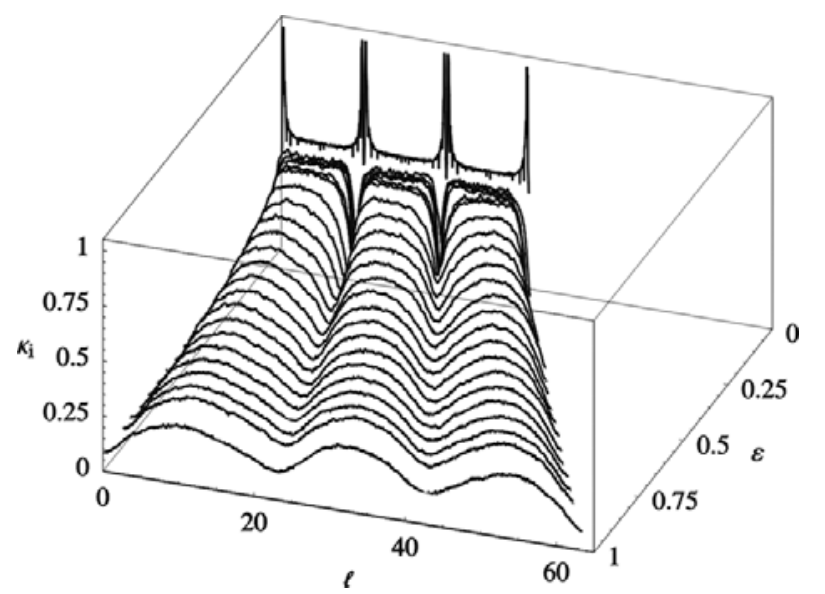

Fig. 6. Curvature within the average shape conformations obtained for knots tied on the rope of increasing length. The first, remarkably different plot was obtained for the initial tightest conformation. The conformation has no freedom to change its shape.

can be described as an almost flat plateau with three valleys. The arc length distances between consecutive valleys are not identical. They can be also used to determine the length of the knot foils. As the knot becomes longer, the valleys become broader and the land between them cannot be considered as a plateau. 


\section{Conclusions and discussion}

Loosening the tightest trefoil knot tied on the hard, floppy rope allows it to fluctuate. The conformation of such a fluctuating knot changes constantly: it is seen not only to change its shape but it seems also to be rotating. The rotation is but an illusion, since tracing the position of a chosen vertex we shall not see it rotating. A direct averaging of the vertices positions would lead in such circumstances to the disappearance of the knot. However, as we have shown, properly designed procedures allow one to remove the apparent rotation and make the consecutive conformations as congruent to each other as possible. Averaging the positions of the vertices of such appropriately oriented and indexed conformations reveals the average shape of the fluctuating knot. The most essential conclusion is that the average shape conformation lacks all symmetry elements characteristic to the tightest trefoil knot. All three foils of the knot are of different length. Since the knot subject to fluctuations was tied on the perfectly floppy, hard rope, the average shape of it can be seen as reference shape for knots tied on ropes of different elastic properties.

Results of our study can be confronted also with results of a recent study performed by Millet et al., see ref. [1]. Knots, that they subject to thermal fluctuations, were also equilateral, but in contrast to our study their six segments were infinitely thin. Thus, their knots were not sensitive to the segment length and their tightest conformation were not well defined. In spite of the essential differences between our studies there is one conclusion that proves to be common: all three axes of the average ellipsoid of inertia are different.

Results presented in this paper are qualitatively different from the results obtained by Saka and Takano [2], who used a model based on a polymer with $N$ segments. This model postulates the existence of two kinds of potentials: the Lennard-Jones potential (repulsive interactions between segments) and a finitely extensible nonlinear elastic potential (attractive interactions between neighboring segments). Our model, based on the perfect rope, does not assume any soft interactions between the vertices or other parts of knots. The only interaction present in our model is the hard interaction which does not allow for existence of overlaps. The average shapes of Saka's knots seem to be symmetrical around the twofold axis passing through the longest foil, while our average conformations do not have this symmetry. Consequently, the two shorter foils of the SK average conformations are of identical length, while the foils of our average conformations are all of different length. It seems improbable to us that the qualitative difference is a result of the different interactions within the SK and our knots. Most probably, the difference stems from imperfections of the shape-fitting procedure applied by Saka and Takano. This needs to be verified.

The work was supported by Poznan University of Technology Grant DS 62-176/11.

Open Access This is an open access article distributed under the terms of the Creative Commons Attribution License (http://creativecommons.org/licenses/by/3.0), which permits unrestricted use, distribution, and reproduction in any medium, provided the original work is properly cited.

\section{References}

1. K.C. Millett, E.J. Rawdon, V.T. Tran, A. Stasiak, J. Chem. Phys. 133, 154113 (2010).

2. S. Saka, H. Takano, J. Phys. Soc. Jpn. 77, 124802 (2008).

3. P. Pieranski, S. Przybyl, Phys. Rev. E 64, 318011 (2001).

4. J. Smutny, Global radii of curvature, and the biarc approximation of space curves: In pursuit of ideal knot shapes, $\mathrm{PhD}$ thesis, École Polytechnique Fédérale de Lausanne (2004).

5. J. Baranska, P. Pieranski, S. Przybyl, E.J. Rawdon, Phys. Rev. E 70, 051810 (2004).

6. W. Kuhn, Kolloid-Z. 68, 2 (1934).

7. E. Guitter, E. Orlandini, J. Phys. A 32, 1359 (1999).

8. V. Katritch, W.K. Olson, A. Vologodskii, J. Dubochet, A. Stasiak, Phys. Rev. E 61, 5545 (2000).

9. R. Metzler, A. Hanke, P.G. Dommersnes, Y. Kantor, M. Kardar, Phys. Rev. Lett. 88, 1881011 (2002).

10. E. Orlandini, A.L. Stella, C. Vanderzande, J. Stat. Phys. 115, 681 (2004).

11. B. Marcone, E. Orlandini, A.L. Stella, F. Zonta, J. Phys. A: Math. Gen. 38, L15 (2005).

12. E. Orlandini, A.L. Stella, C. Vanderzande, Phys. Biol. 6, 025012 (2009).

13. E. Ben-Naim, Z.A. Daya, P. Vorobieff, R.E. Ecke, Phys. Rev. Lett. 86, 1414 (2001).

14. P.G. Dommersnes, Y. Kantor, M. Kardar, Phys. Rev. E 66, 031802 (2002).

15. A. Amzallag, C. Vaillant, M. Jacob, M. Unser, J. Bednar, J. Kahn, J. Dubochet, A. Stasiak, J.H. Maddocks, Nucl. Acids Res. 34, e125 (2006). 